\title{
Pilot study for the presence of fungal metabolites in sheep milk from first spring milking
}

\author{
Marta Piątkowska ${ }^{1}$, Michael Sulyok ${ }^{2}$, \\ Katarzyna Pietruszka ${ }^{1}$, Łukasz Panasiuk ${ }^{1}$ \\ ${ }^{1}$ Department of Pharmacology and Toxicology, \\ National Veterinary Research Institute, 24-100 Pulawy, Poland \\ ${ }^{2}$ Center for Analytical Chemistry, Department of Agrobiotechnology (IFA-Tulln), \\ University of Natural Resources and Life Sciences, Vienna (BOKU), A-3430 Tulln, Austria \\ marta.piatkowska@boku.ac.at
}

Received: March 21, 2018

Accepted: June 7, 2018

\begin{abstract}
Introduction: A mini-study of 20 raw milk samples was conducted to examine the spectrum of fungal metabolites in sheep milk from the first spring milking. Material and Methods: Samples were collected from randomly selected ewes in two animal flocks from the Bieszczady Mountains and analysed using liquid chromatography-tandem mass spectrometry. Results: Out of $\sim 700$ bacterial, fungal, and plant metabolites tested for, only one mycotoxin - Enniatin B - was detected in sheep milk samples $(18 / 20 ; 0.0055-0.0121 \mu \mathrm{g} / \mathrm{kg} ; 0.0078 \mu \mathrm{g} / \mathrm{kg}$ average). Conclusions: The results indicated that there was no high-level exposure to fungal metabolites via consumption of raw sheep milk during the sample collection period.
\end{abstract}

Keywords: sheep milk, mycotoxins, fungal metabolites, LC-MS/MS.

\section{Introduction}

Mycotoxins are low molecular weight secondary metabolites of fungi of different genera, e.g. Aspergillus, Alternaria, Fusarium, and Penicillium. Mycotoxin production can occur under favourable conditions of humidity, temperature, and level of oxygen in the field, during harvesting, or postharvesting during transport or storage. The presence of toxic metabolites in an animal diet may cause many serious health conditions such as teratogenesis, carcinogenesis, kidney and liver damage, or immune system suppression. The toxic effects differ by animal species and depend on many factors, but chronic exposure may lead to toxicity even at low levels (12). Although currently there are about 100,000 formally described fungi species, only a limited number of them exhibit toxicological effects in humans and animals, and are subject to legal regulation (20). At present, there are regulations for the presence of 8 mycotoxins in feed (aflatoxin B1, fumonisins B1 and B2, ochratoxin, zearalenone, T-2 and HT-2 toxins, and deoxynivalenol) $(5,7,9,16)$. In terms of the present study a very important regulation is
1881/2006 (8) for the presence of aflatoxin M1 in milk and milk products $(0.05 \mu \mathrm{g} / \mathrm{kg})$, infant formulae, and dietary foods for special medical purposes and intended specifically for infants $(0.025 \mu \mathrm{g} / \mathrm{kg})$. For many other mycotoxins setting toxicological thresholds is not possible due to the lack of data on occurrence and hence exposure (4). Additionally, full evaluation of toxic effect is not possible due to the co-occurrence of mycotoxins and mycotoxin co-occurrence with other metabolites of bacterial or plant origin, e.g. ergot alkaloids or lolitrem $\mathrm{B}$. These alkaloids may also cause toxic effects in animals, resulting in a reduction of body weight, increased temperature, impaired reproduction and milk production, and decreased blood flow (17). Their presence is also associated with fescue toxicosis, a disease occurring in sheep and cattle causing gangrene, swelling of the limbs, or hyperthermia.

Although many experiments have been conducted to study the carry-over of mycotoxins/metabolites from different feed matrices to ruminant milk, the studies were performed using levels well above the set maximum limits (22). Additionally, in nature, the animals are exposed to intake of a "cocktail" of 
different contaminants, coming from their varied diet. There are still data gaps for the proper evaluation of mycotoxin or metabolites transfer from diet to animal milk, and consequently for the evaluation of consumer exposure to low levels of natural toxins. Although the European Commission recommends their member states to survey for multi-contaminant presence so that co-occurrence can be assessed, only a few studies have been conducted to study the co-occurrence of mycotoxins. Their synergistic, antagonistic, and additive inhibitory effects are still unknown. With proper multi-residue analytical methods these effects could be studied and better risk assessment could be made. For now, aflatoxin M1 remains the most studied mycotoxin in milk, with only a few studies focused on other mycotoxins (12).

The study described in this paper was designed to define the potential risk to consumers in local products from the Polish Bieszczady Mountains made from unprocessed sheep milk contaminated with mycotoxins. There are several reasons why this study focuses on sheep milk. First, the history of shepherdry in Poland goes back to the $11^{\text {th }}$ century, and pasturing and sheep milk processing were the main occupations of highlanders (18). Traditional pasturing practice also requires the manufacturing of traditional sheep milk products according to long-established techniques (13). Among the products, a cheese called "oscypek" is the best known example of regional food in Poland. Additionally, oscypek cheese represents high economic value, constituting the basis of a large and diversified production and retail sector. It is important that the cheese is handmade from nonpasteurised milk in premises on the mountain according to the recipe passed on from generation to generation in an unwritten form, which makes the final product unique to each shepherd who makes it (14). Nowadays oscypek is a popular tourist souvenir. Another reason to conduct the study is that the possible level of contamination of sheep milk in Poland with such a wide range of analytes has not been studied so far.

\section{Material and Methods}

Sampling of sheep milk. A total of 20 randomly selected ewes in two different flocks of sheep ( 50 animals) were milked for samples (10 samples per flock). The animals were kept in the Bieszczady Mountains in Southeastern Poland (Fig. 1). The sampling began on the first day of animal grazing, in April. Samples $(50 \mathrm{ml})$ were immediately frozen at $-18^{\circ} \mathrm{C}$ and sent to the Reference Laboratory for Mycotoxin Analysis, National Veterinary Research Institute (NVRI), Pulawy, Poland to be tested. They were stored for a maximum of two weeks before submission for analysis.

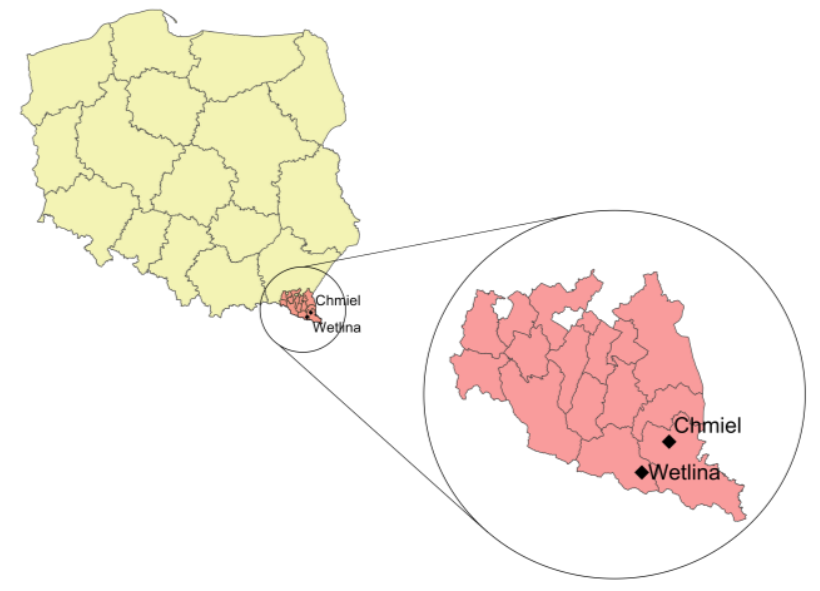

Fig. 1. Geographical location of the sheep flocks selected for the analysis

Chemicals and reagents. Acetonitrile (ACN), HPLC grade methanol $(\mathrm{MeOH})(99.5 \%)$, and acetic acid were provided by J.T. Baker (USA). Ammonium acetate (LC/MS grade) and chloroform were supplied by Sigma-Aldrich (Germany). Water was purified through a Milli-Q plus system from Millipore (USA). A working standard solution of bacterial, fungal, and plant metabolites was obtained as a gift from the research group of IFA-Tulln, stored at $-18^{\circ} \mathrm{C}$, and used for the preparation of calibration curves and spiking experiments. Standards of fumonisins B1 and B2 and aflatoxins M1 (AFL M1) and M1 U- ${ }^{13} \mathrm{C}_{17}$ (AFL M1-IS) were obtained from Sigma-Aldrich (Germany). The stock solutions of fumonisins were prepared in a $50 \%$ solution of $\mathrm{ACN}$ in $\mathrm{H}_{2} \mathrm{O}$ while stock solutions of AFL M1 and AFL M1-IS were prepared in chloroform and stored at $-18^{\circ} \mathrm{C}$ prior to analysis.

Sample preparation. Sample pretreatment procedure for the multi-content analysis was adopted from Malachova et al. (19). Briefly, $5.0 \mathrm{~mL}$ of milk sample was weighed into a $50 \mathrm{~mL}$ Falcon plastic tube and $20 \mathrm{~mL}$ of extraction solvent (acetonitrile/water/acetic acid 79:20:1, v/v/v) was added. The samples were extracted for $90 \mathrm{~min}$ using a vertical shaker (200 cycles/min) and subsequently centrifuged (3,500 rpm, $5 \mathrm{~min})$. Afterwards, $500 \mu \mathrm{L}$ of the extracts were transferred into glass vials and diluted with the same volume of dilution solvent (acetonitrile/water/acetic acid 20:79:1, v/v/v). After vortexing, $5 \mu \mathrm{L}$ of the diluted extracts were injected into the LC-MS/MS system without further pretreatment.

For the screening of the samples for AFL M1 contamination, $25 \mathrm{~mL}$ of milk sample was centrifuged (3,500 rpm, $15 \mathrm{~min})$ and the upper layer of fat was discarded. A further $10 \mu \mathrm{L}$ of AFL M1-IS was added, samples were vortexed, and $25 \mathrm{~mL}$ of acetonitrile and ready Q-sep QuEChERS Extraction Salts (Agilent, USA) were added for extraction. Samples were vortexed for $2 \mathrm{~min}$, centrifuged (3,500 rpm, $15 \mathrm{~min})$ 
and the organic layer was transferred to a fresh conical flask for rotary evaporation $\left(40 \pm 5^{\circ} \mathrm{C}\right)$. The dry residues were reconstituted with $10 \mathrm{~mL}$ of $20 \%$ methanol and loaded into Afla M1 HPLC immunoaffinity columns (Vicam, USA). The columns were rinsed with $10 \mathrm{~mL}$ of water, dried, and the analyte was eluted with 2 and $1 \mathrm{~mL}$ of acetonitrile subsequently. Extracts were evaporated under a gentle stream of nitrogen at $40 \pm 5^{\circ} \mathrm{C}$, the dry residues were reconstituted with $120 \mu \mathrm{L}$ of mobile phase $\mathrm{A}$ and $120 \mu \mathrm{L}$ of mobile phase $\mathrm{B}$, and $5 \mu \mathrm{L}$ was injected into the LC-MS/MS system for analysis.

Spiking experiments and preparation of calibration curves. For the spiking experiments for multi-content analysis the sample preparation procedure was miniaturised in order to decrease the amount of standards needed. An amount of $0.25 \mathrm{mg}$ of milk sample was weighed into glass vials and evaporated to dryness. A further $100 \mu \mathrm{L}$ of working standard solution was added and samples were extracted using $900 \mu \mathrm{L}$ of extraction solvent. After the extraction, the samples were vortexed and $500 \mu \mathrm{L}$ of the extracts was diluted with the same volume of dilution solvent. An external calibration curve was prepared by dilution of appropriate amounts of the final working solution with acetonitrile/water/acetic acid (49.5/49.5/1, v/v/v) at levels corresponding to 3, 10, 30, and 100-fold dilution of the fortification level (given in Table 1) plus two points at 3 and 10 times the fortification level to ensure that all spiking levels fell into the calibration range.

For the analysis of AFL M1 a four-point matrixmatched calibration curve was prepared at the levels corresponding to $0,0.5,1$, and 2 spiking level sets as $50 \mathrm{ng} / \mathrm{kg}$. The sample fortified at the level of $50 \mathrm{ng} / \mathrm{kg}$ was prepared in two replicates to ensure the internal quality control.

Instrumental parameters. The LC-MS/MS system for the analysis of $\sim 700$ bacterial, fungal, and plant metabolites consisted of an Agilent 1200 series liquid chromatograph (Agilent Technologies, Germany) and a QTRAP 5500 triple quadrupole mass analyser (Sciex, Canada) controlled by Analyst 1.6.2 software (Sciex). Analytes were separated using a Gemini $\mathrm{C}_{18}$-column, $150 \times 4.6 \mathrm{~mm}$ i.d., $5 \mu \mathrm{m}$ particle size, operated at $25^{\circ} \mathrm{C}$, and equipped with a security guard cartridge of the same material (Phenomenex, USA). Elution was carried out in a gradient mode with the flow rate set as $1,000 \mu \mathrm{L} / \mathrm{min}$. using a mobile phase consisting of methanol/water/acetic acid 10:89:1 (v/v/v; eluent A) and 97:2:1 (v/v/v; eluent B), both containing $5 \mathrm{mM}$ of ammonium acetate. ESI-MS/MS was performed in the scheduled selected reaction monitoring (sSRM) mode both in positive $\left(\mathrm{ESI}^{+}\right)$and negative $\left(\mathrm{ESI}^{-}\right)$polarities in two separate chromatographic runs. This method was transferred from the Department of Agrobiotechnology, Tulln,
Austria (IFA-Tulln) to the NVRI, Pulawy, Poland, in order to conduct this study.

For the analysis of AFL M1, a Nexera X2 system was used coupled with LCMS-8050 triple quadrupole mass spectrometer (Shimadzu, Japan) equipped with an electrospray operated in positive $\left(\mathrm{ESI}^{+}\right)$mode and controlled by LabSolution software (Shimadzu). Chromatographic separation was performed on a Kinetex BiPhenyl column, $100 \times 2.1 \mathrm{~mm}$ i.d., $2.6 \mu \mathrm{m}$ particle size coupled with a BiPhenyl security guard cartridge (Phenomenex, USA). The column oven temperature was set at $40^{\circ} \mathrm{C}$. The separation was performed using a gradient elution of the mobile phase consisting of $1 \mathrm{mM}$ of ammonium acetate and $0.1 \%$ of acetic acid/MeOH 95:5 (v/v; eluent $\mathrm{A})$ and of $1 \mathrm{mM}$ ammonium acetate and $0.1 \%$ of acetic acid/MeOH 5:95 (v/v; eluent B).

Data evaluation. MultiQuant ${ }^{\mathrm{TM}}$ 2.0.2 software (Sciex, USA) and LabSolution were used for the peak integration and preparation of linear $1 / x$ weighted calibration curves obtained from the analysis of neat solvents and spiked samples to evaluate the linearity of the method.

The qualitative analysis was performed by comparing the peak retention time in the chromatogram of the analysed sample with the peak retention times in the chromatograms of the samples of the calibration curve and the spiked sample. Limits of detection and quantification were calculated based on a signal-to-noise ratio of blank samples (LOD $\mathrm{S} / \mathrm{N}=3$, LOQ $\mathrm{S} / \mathrm{N}=6$ ).

\section{Results}

The results indicated that samples were contaminated with one fungal metabolite, Enniatin B, at a relatively low level of $0.0121 \mu \mathrm{g} / \mathrm{kg}$ or below (Table 1), and no other fungi, plant, or bacterial metabolites were found. A statistical presentation of Enniatin B detected in raw sheep milk on the first day of pasturing is made in Fig. 2.

Table 1. Individual concentrations of Enniatin B $(\mu \mathrm{g} / \mathrm{kg})$ detected in two tested flocks

\begin{tabular}{lll}
\hline & Flock 1 & Flock 2 \\
\hline Sample 1 & 0.0085 & $-^{*}$ \\
Sample 2 & 0.0074 & 0.0095 \\
Sample 3 & 0.0079 & 0.0063 \\
Sample 4 & 0.0082 & 0.0055 \\
Sample 5 & 0.0067 & 0.0058 \\
Sample 6 & 0.0075 & $-*$ \\
Sample 7 & 0.0085 & 0.0102 \\
Sample 8 & 0.0074 & $<$ LOQ** \\
Sample 9 & 0.0078 & 0.0066 \\
Sample 10 & 0.0077 & 0.0121 \\
\hline
\end{tabular}

* - not detected above the limit of detection (LOD); ** - detected between the LOD and level of quantification (LOQ) 


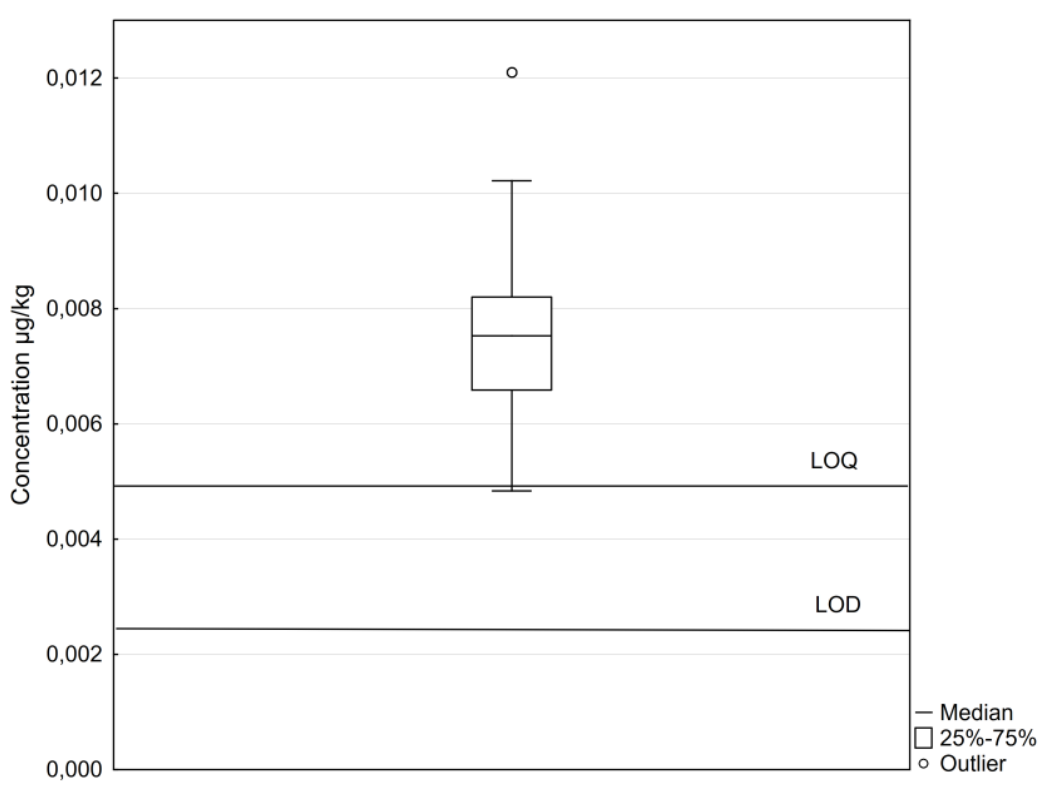

Fig. 2. Statistical presentation of detected concentrations of analytes

Table 2. Method performance characteristics $(n=3)$ and detected concentrations of Enniatin B. Results were corrected for the recoveries

\begin{tabular}{|c|c|c|c|c|c|c|c|}
\hline \multirow{2}{*}{ Analyte } & \multirow{2}{*}{$\begin{array}{l}\text { Fortification } \\
\text { level }(\mu \mathrm{g} / \mathrm{kg})\end{array}$} & \multirow{2}{*}{$\begin{array}{l}\text { Apparent } \\
\text { recovery } \\
(\%)(\mathrm{RSD})\end{array}$} & \multirow{2}{*}{$\begin{array}{l}\text { LOD } \\
(\mu \mathrm{g} / \mathrm{kg})\end{array}$} & \multirow{2}{*}{$\begin{array}{l}\mathrm{LOQ} \\
(\mu \mathrm{g} / \mathrm{kg})\end{array}$} & \multicolumn{3}{|c|}{ Concentrations detected $(\mu \mathrm{g} / \mathrm{kg})$} \\
\hline & & & & & Min. & Max. & Average \\
\hline Aflatoxin M1 & 0.05 & $86.2(9.9)^{*}$ & 0.0044 & 0.0088 & - & - & - \\
\hline Enniatin B & 0.1545 & $74.4(3.3)$ & 0.0025 & 0.0049 & 0.0055 & 0.0121 & 0.0078 \\
\hline
\end{tabular}

* Extraction efficiency $\mathrm{R}_{\mathrm{E}}(\%)$

\section{Discussion}

Method transfer. In the field of multicontaminant analysis there is a tendency towards analytical methods using liquid chromatography mass spectrometry with a simple and cheap sample preparation step (19). A multi-residue, easy, and reliable analytical method for the simultaneous determination of bacterial, fungal, and plant metabolites was therefore successfully transferred from the Department of Agrobiotechnology (IFA-Tulln), in Tulln, Austria, to the NVRI in Pulawy, Poland. After adjusting the method for different equipment its performance characteristics were calculated and the method was used to screen the milk samples.

A transfer of the acquisition method from the QTrap 5500 Sciex instrument from IFA-Tulln to an equivalent instrument at the NVRI was the first step of the study. It was a method developed by Malachova et al. (19) which was extended to a wide range of additional bacterial, fungal, and plant metabolites (24).

The sample preparation procedure was kept largely as devised at IFA. The only difference was that after the extraction milk samples were centrifuged for $5 \mathrm{~min}$ at $3,500 \mathrm{rpm}$ instead of being left for gravitational settlement for $5 \mathrm{~min}$.

Determination of method performance characteristics. As the multi-residue method used for examination of possible sheep milk contamination with naturally occurring toxins was not previously used for this purpose, there were no validation parameters available (currently the method is validated for $\sim 500$ analytes in figs, maize, and nuts, data unpublished). To set the method's LOD, LOQ, and apparent recoveries $\left(\mathrm{R}_{\mathrm{A}}, \%\right)$ as needed for result interpretation and correction, three spiked sheep milk samples were subjected to analysis in order to conduct the mini survey. The samples were spiked with the working standard mix containing 700 analytes and the method parameters were calculated for the analytes of interest. We decided to spike only three samples due to the limited amount of standard and its high cost. The full validation of the method was not the aim of the study.

The procedure used for the determination of AFL M1 content in milk was adopted from the National Control Programme for Poland, conducted at the NVRI, Pulawy, Poland. The full validation had already been performed according to the Commission Decision 2002/657/EC (6) and the method characteristics like LOD, LOQ, and extraction efficiency $\mathrm{R}_{\mathrm{E}}(\%)$ for detected analytes using both methods are given in Table 2.

A mini survey for the presence of fungal metabolites in sheep milk. Ruminants are more resistant to mycotoxins than monogastric animals, as the rumen microbes can degrade or metabolise some 
mycotoxins. Despite this barrier, the surveys of the mycotoxins in ruminants' milk demonstrate that under special conditions the presence of fungal metabolites in milk is possible (11). Even if the presence of AFL M1 has been of the most concern in milk since the 1960s, many other mycotoxins have been found in grains, feeds, and especially silage (personal experience). One of the groups of mycotoxins to cause concern are the trichothecenes, which are modified forms of mycotoxins produced by Fusarium species. This group includes among others, T-2 and HT-2 toxins, deacetoxyscirpenol (DAS), deoxynivalenol (DON), 3-acetyldeoxynivalenol (3-AcDON), 15-acetyldeoxynivalenol (15-AcDON), and ruminant metabolite deepoxy-deoxynivalenol (DOM-1), fumonisins (FUMs, FB1, FB2, FB3), zearalenone $(\mathrm{ZEN})$, which is metabolised in ruminants to $\alpha$-zearalenol ( $\alpha$-ZEL) and $\beta$-zearalenol ( $\beta$-ZEL), $\alpha$-zearalanol (zeranol) ( $\alpha$-ZAL), and $\beta$-zearalanol (taleranol) ( $\beta$-ZAL) (23). Due to possible contamination with a huge diversity of metabolites (20) and subsequent risk of metabolite carry-over to milk, we decided to screen the sheep milk for the presence of all the metabolites currently included in the method.

Enniatins (ENNs) A, A1, B, and B1 are structurally related mycotoxins produced by various Fusarium species which invade and grow on crops and may produce these toxins under moist and cool conditions. They occur as contaminants mainly in cereals, such as wheat, barley, and maize, but also in oats, rye, and rice (25). ENNs are called "emerging mycotoxins" as their occurrence is progressively increasing in food and feed and there is great concern about their presence worldwide (26). The data submitted to EFSA by 12 European countries indicated a high correlation between the four enniatins in cereal grain samples collected between 2000 and 2013 (4). It is also expected that they may co-occur with other Fusarium metabolites, like deoxynivalenol (DON), moniliformin (MON), and fumonisins (FUMs).

In the current study, only one of the ENNs A, A1, $\mathrm{B}$, and $\mathrm{B} 2$ was detected in raw sheep milk. The presence of ENN B was determined in 18 out of 20 tested samples, with the average concentration of $0.0078 \pm 0.0017 \mu \mathrm{g} / \mathrm{kg}$. No data on the presence of the ENNs in raw sheep milk are available for comparison. In general, sheep are considered to be the most resistant ruminants to mycotoxins (27). On the other hand, although the rumen microbiota can degrade mycotoxins, some of them exert strong antimicrobial activity (e.g. enniatins, beauvericin) and thus may modify the rumen flora. If the degrading capacity is diminished, it may lead to unexpected passage of toxins (11). Even though the transfer of mycotoxins to milk is generally low, the blood-milk barrier may be also affected, e.g. by changed $\mathrm{pH}$ relationship between blood plasma and milk. In a healthy animal, the $\mathrm{pH}$ of milk is lower than the plasma $\mathrm{pH}$, while in a diseased animal the $\mathrm{pH}$ of milk equals or exceeds the blood plasma $\mathrm{pH}$. This may modulate the rate of transfer and facilitate the excretion of mycotoxins that are not expected in milk.

In the literature, the most studied mycotoxin in milk is AFL M1, which has the highest potential to be carried over to milk. It should be detectable in sheep milk $6 \mathrm{~h}$ from the first consumption of aflatoxin B1 (AFL B1)-contaminated feed (21), and this route of excretion is the only one possible. Human exposure to this toxin via the consumption of contaminated milk of different ruminant species has also been widely studied. In raw sheep milk surveys conducted during the recent years have demonstrated AFL M1 presence with contamination and concentration depending on the geographical location (e.g. at the level of $28.1 \pm$ $13.7 \mathrm{ng} / \mathrm{L}$ in Iran in $2010(22), 118.6 \pm 114.2 \mathrm{ng} / \mathrm{L}$ in Croatia (goat and sheep raw milk) in 2013 (2), $3.33 \pm$ $0.09 \mathrm{ng} / \mathrm{L}$ in Lebanon in 2014 (15), and $5.11 \pm$ $0.21 \mathrm{ng} / \mathrm{kg}$ in Croatia, in 2016) (3). Moreover, in Iran in 2011 (10) and 2016 (1), 25\% and 26.9\% of the samples, respectively exceeded the EU limit set at $50 \mathrm{ng} / \mathrm{kg}$. In the current study the presence of AFL M1 in raw sheep milk was not detected, which concurs with the results of the National Control Programme in Poland. This is due to two reasons, the first of which is - climatic conditions in Poland are not favourable to AFL B1, and the second is that - an efficiently functioning feed control programme prevents highly contaminated feeds from entering the market.

Summing up, in Poland, there was no high level exposure to fungal metabolites jeopardising consumers of sheep milk during the sample collection period.

Conflict of Interests Statement: The authors declare that there is no conflict of interests regarding the publication of this article.

Financial Disclosure Statement: The study was funded by the KNOW (Leading National Research Centre) Scientific Consortium "Healthy Animal - Safe Food", Ministry of Science and Higher Education Resolution No. 05-1/KNOW2/2015.

Animal Rights Statement: None required.

Acknowledgments: The Authors are immensely grateful to Damian Jus and Stanislaw Staszel for animal sample collection.

\section{References}

1. Bahrami R., Shahbazi Y., Nikousefat Z.: Aflatoxin M1 in milk and traditional dairy products from west part of Iran: occurrence and seasonal variation with an emphasis on risk assessment of human exposure. Food Control 2016, 62, 250-256, doi:10.1016/j.foodcont.2015.10.039.

2. Bilandžić N., Božić D., Dokić M., Sedaka M., Solomun Kolanović B., Varenina I., Tanković S., Cvetnić Ž.: Seasonal effect on aflatoxin M1 contamination in raw and UHT milk from 
Croatia. Food Control 2014, 40 260-264, doi: 10.1016/j.foodcont.2013.12.002.

3. Bilandžić N., Varenina I., Solomun Kolanović B., Božić Luburić Đ., Varga I., Želježić B., Cvetnić L., Benić M., Tanković S., Cvetnić Ž.: Occurrence of aflatoxin M1 in raw cow, goat, and sheep milk during spring and autumn in Croatia during 2016. Toxin Rev 2017, 36, 290-296, doi: 10.1080/15569543.2017.1306785.

4. EFSA. Scientific opinion on the risks to human and animal health related to the presence of beauvericin and enniatins in food and feed. EFSA J 2014, 12, 3595. https://www.efsa.europa.eu/en/efsajournal/pub/3802 (accessed on 14.01.2018).

5. European Commission. Commission Recommendations 2013/165/EC of 27 March on the presence of T-2 and HT-2 toxin in cereals and cereal products. Off J Eur Union 2013, L 91, 12 15. http://eur-lex.europa.eu/legal-content/EN/TXT/?uri=CELEX \%3A32013H0165 (accessed on 13.01.2018).

6. European Commission. Commission Decision of 12 August 2002 implementing Council Directive 96/23/EC concerning the performance of analytical methods and the interpretation of results. Off J Eur Communities 2002, 221, 8-36. http://eurlex.europa.eu/legal-content/EN/TXT/?uri=CELEX\%3A32002D0 657 (accessed on 13.01.2018).

7. European Commission. Commission Recommendation 2006/576/EC of 17 August 2006 on the presence of deoxynivalenol, zearalenone, ochratoxin A, T-2 and HT-2 and fumonisins in products intended for animal feeding. Off. J Eur Union 2006, L229, 7-9.

8. European Commission. Commission Regulation (EC) No 1881/2006 of 19 December 2006 setting maximum levels for certain contaminants in foodstuffs. Off J Eur Union 2006, L364, 5-24. http://eur-lex.europa.eu/legal-content/EN/ALL /?uri=CELEX\%3A32006R1881 (accessed on 13.01.2018).

9. European Parliament and the Council of the EU. Directive of the European Parliament and of the Council of 7 May 2002 on undesirable substances in animal feed 2002/32. Off J Eur Communities, 2002, 1-15. http://eur-lex.europa.eu/legalcontent/EN/TXT/?uri=celex\%3A32002L0032 (accessed on 13.01.2018)

10. Fallah A., Fazlollahi R., Emami A.: Seasonal study of aflatoxin M1 contamination in milk of four dairy species in Yazd, Iran. Food Control 2016, 68, 77-82.

11. Fink-Gremmels J.: Mycotoxins in cattle feeds and carryover to dairy milk: a review. Food Addit Contam A 2008, 25, 172-180.

12. Flores-Flores M.E., Lizarraga E., Lopez de Cerain A., GonzalezPenas E.: Presence of mycotoxins in animal milk: a review. Food Control 2015, 53, 163-176.

13. Gasienica-Chmiel M.: Preservation of the cultural heritage of the Tatra National Park as exemplified by pasturing (in Polish). Doctoral thesis, Warsaw University of Life Sciences - SGGW, Warsaw, Poland, 2001

14. Gorlach K., Starosta P., Pilichowski A., Adamski T., Dzwonkowska K.: Local food production and knowledge dynamics in rural sustainable development in Poland. In: Local Food Production, Non-agricultural Economies and Knowledge Dynamics in the Rural Sustainable Development (Czech, Hungarian, and Polish cases); edited by Gorlach K., Kovách I., Political Science Institute of the Hungarian Academy of Sciences, Budapest, Hungary 2006, pp. 38-54,

15. Hassan H.F., Kassaify Z.: The risks associated with aflatoxins M1 occurrence in Lebanese dairy products. Food Control 2014, $37,68-72$.

16. Iheshiulor O.O.M., Esonu B.O., Chuwuka O.K., Omede A.A., Okoli I.C., Ogbuewu I.P.: Effects of mycotoxins in animal nutrition: a review. Asian J Anim Sci 2011, 5, 19-33.

17. Koczwara K., Pańka D., Lisiecki K., Juda M.: The possibility of use of endophytes in biological plant protection. J Educ Health Sport 2015, 5, 333-340. doi: 10.5281/zenodo.18696.

18. Kuźnicka E., Rant W., Radzik-Rant A.: Adversities and opportunities in the production of "Oscypek" cheese and in the traditional grazing system of Polish Mountain Sheep. Options Méditerranéennes 2008, 38, 405-407.

19. Malachová A., Sulyok M., Beltrán E., Berthiller F., Krska R.: Optimization and validation of a quantitative liquid chromatography-tandem mass spectrometric method covering 295 bacterial and fungal metabolites including all regulated mycotoxins in four model food matrices. J Chrom A 2014, 1362, 145-156.

20. Malachová A., Stránská M., Václavíková M., Elliott C.T., Black C., Meneely J., Hajšlová J., Ezekiel C.N., Schuhmacher R., Krska R.: Advanced LC-MS-based methods to study the cooccurrence and metabolization of multiple mycotoxins in cereals and cereal-based food. Anal Bioanal Chem 2017, 410, 801-825.

21. Pittet A.: Natural occurrence of mycotoxins in foods and feeds an updated review. Rev Med Vet 1998, 149, 479-492.

22. Rahimi E., Bonyadian M., Rafei M., Kazemeini H.R.: Occurrence of aflatoxin M1 in raw milk of five dairy species in Ahvaz, Iran. Food Chem Toxicol 2010, 48, 129-131.

23. Sørensen L.K., Elbæk T.H.: Determination of mycotoxins in bovine milk by liquid chromatography tandem mass spectrometry. J Chrom B 2005, 820, 183-196.

24. Sulyok M., Steiner D., Krska R.: Analytical performance of an LC-MS/MS based multi-class method covering > 1000 analytes. $8^{\text {th }}$ International Symposium on Recent Advances in Food Analysis; November 7-10, 2017, Prague, Czech Republic.

25. Tsiplakou E., Anagnostopoulos C., Liapis K., Haroutounian S.A., Zervas, G.: Determination of mycotoxins in feedstuffs and ruminant's milk using an easy and simple LC-MS/MS multiresidue method. Talanta 2014, 130, 8-19.

26. Yoshinari T., Suzuki Y., Sugita-Konishi Y., Ohnishi T., Terajima J.: Occurrence of beauvericin and enniatins in wheat flour and corn grits on the Japanese market, and their cocontamination with type B trichothecene mycotoxins. Food Addit Contam A 2016, 33, 1620-1626.

27. Zain M.E.: Impact of mycotoxins on humans and animals. J Saudi Chem Soc 2011, 15, 129-144. 